\title{
SYSTEMATIC ERRORS OF THE CRIMEAN VECTOR MAGNETOGRAPH
}

\author{
V. A. KOTOV
}

Crimean Astrophysical Observatory, Crimea, U.S.S.R.

\begin{abstract}
The influence of some 'effective' asymmetry (miscentering) of spectral line on the accuracy of the complete field vector is considered for the measurements with the Crimean magnetograph. This miscentering appears in the device for $H_{\perp}$ records if the longitudinal field is strong.
\end{abstract}

In several papers (Kuznecov et al., 1966; Beckers, 1968) it has been pointed out that the transversal field-mode of magnetographs similar to the Crimean one possesses a systematic error connected with the influence of the longitudinal field on the line of sight velocity signal. Namely when the phase-difference produced by the $A D P$ crystal vanishes, the electro-optic light modulator (EOM) works as an analyser of circularpolarized light. Recently Wiehr (1969) has considered this effect for the Locarno magnetograph and for one of the modes of the Crimean magnetograph (when the optical axes of $\lambda / 4$-plate and $A D P$ coincide). But the detailed analysis of possible errors in the determination of $H_{\|}, H_{\perp}$ field components, inclination $\gamma$ and azimuth $\chi$

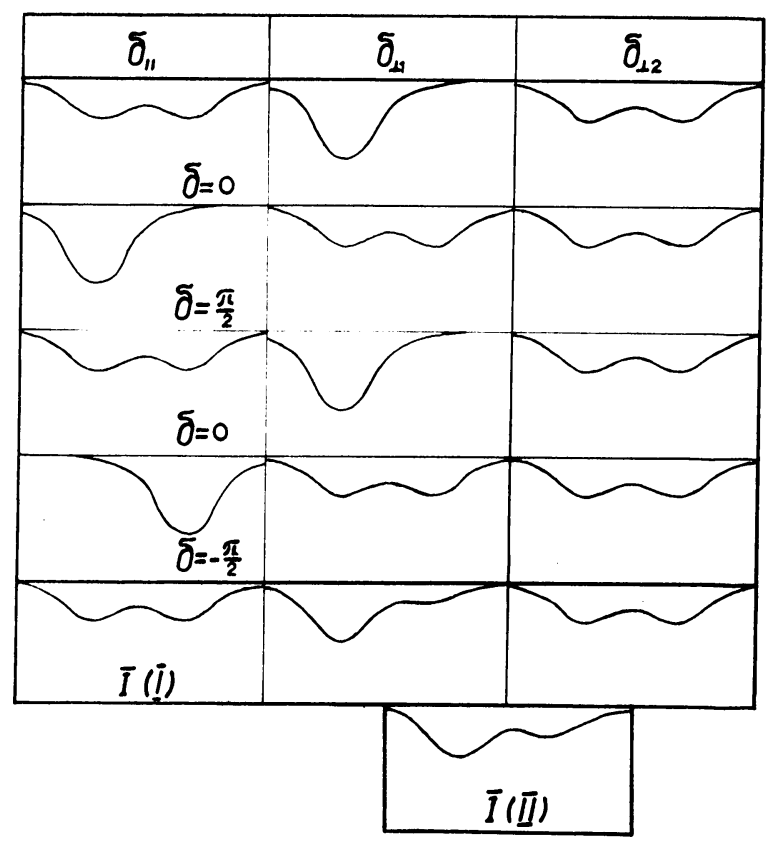

Fig. 1. The line profiles produced by the EOM at different phase-differences $\delta$ of crystal in different types of light modulation $\left(\delta_{\|}, \delta_{\perp 1}, \delta_{\perp 2}\right)$. The line profiles $I$ average in time for modes $I$ and $I I$ are shown at the bottom of the picture. 
has not been performed. We have also estimated these errors in the new variant of the Crimean magnetograph (Nikulin, 1967).

The Crimean magnetograph gives two possibilities to measure the total magnetic vector: the mode $(I)$ for successive separate recording of three field components, described by Stepanov and Severny (1962), and another mode (II) for simultaneous recording (Nikulin, 1967). Figure 1 presents schematically the line profiles produced by the EOM at different phase-differences $\delta$ produced by the $A D P$ crystal. We see asymmetry of the line profile $I$ average in time (at the bottom of the picture) for the second mode; in mode $I$ the asymmetry appears only for $\delta_{\perp 1}$ recording.

To examine this effect we have calculated a fictitious velocity, due to asymmetry, and magnetic signals for Fe I $\lambda 5250$ line using Unno (1956) theory. This velocity signal upon the field strength $H$ and inclination $\gamma$ is plotted in Figure 2. The dotted and solid lines refer to modes $I$ and $I I$ respectively. We see that fictitious velocities can reach $3-5 \mathrm{~km} / \mathrm{s}$ for $H \gtrsim 1500 \mathrm{G}$, especially for mode $I$ (separate recording of $\delta_{\perp 1}$ signal). In this case the line-shift is twice as much as it is in mode $I I$ for $H \lesssim 1000 \mathrm{G}$ and for small values of $\gamma$.

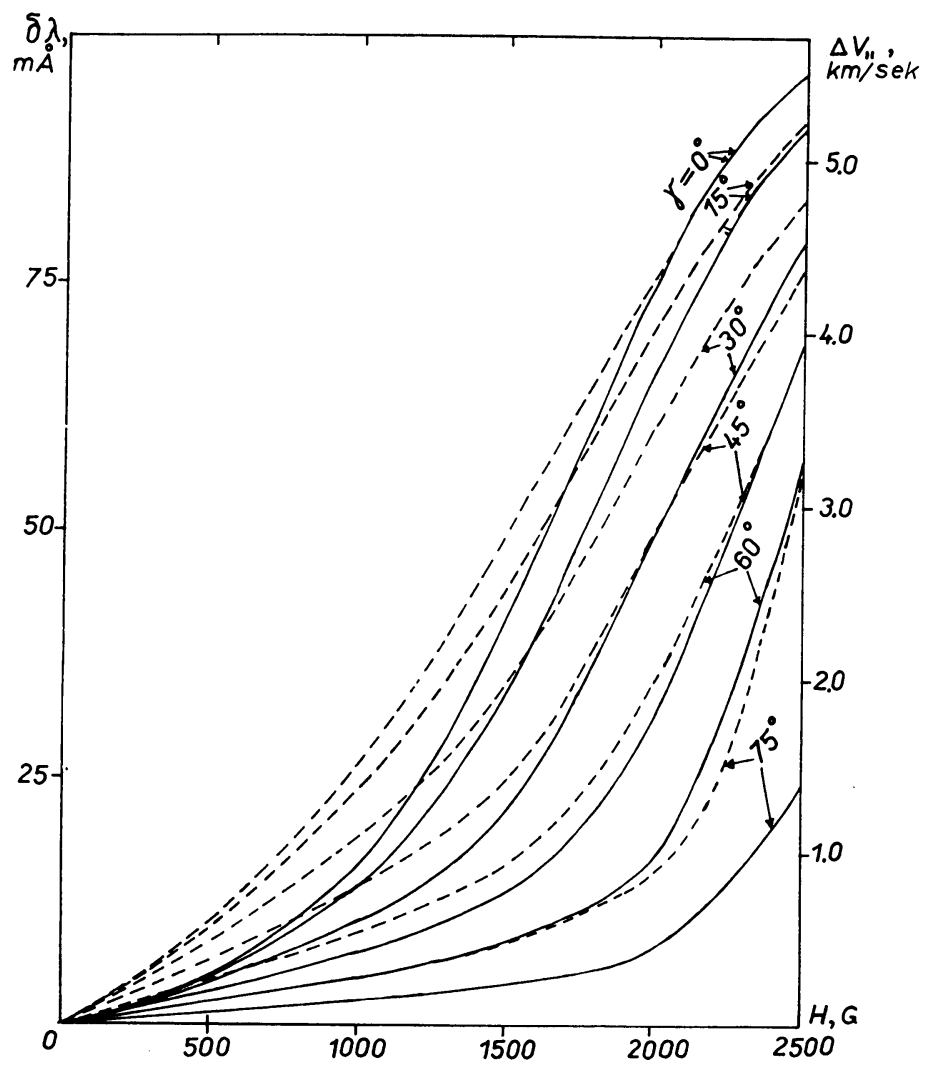

Fig. 2. The dependence of the line shift produced by asymmetry of the line profile upon field strength $H$ and inclination $\gamma$. 
The dependence of the ratio $\delta_{\perp} / \delta_{\perp}^{0}\left(\delta_{\perp}^{0}=\sqrt{ }\left\{\left(\delta_{\perp 1}^{0}\right)^{2}+\left(\delta_{\perp 2}^{0}\right)^{2}\right\}\right.$ is the undistorted value) on $H$ and $\gamma$ for mode $I I$ is illustrated in Figure 3. The errors may be quite appreciable for strong $H_{\|}$, but when $\gamma \gtrsim 45^{\circ}$ and $H \lesssim 1500 \mathrm{G}$ the errors do not exceed $12 \%$; for $H \lesssim 1000 \mathrm{G}$ they are less than $18 \%$ for any value of $\gamma$. These errors are well within the limits of the usual accuracy of magnetograph measurements. As may be realized

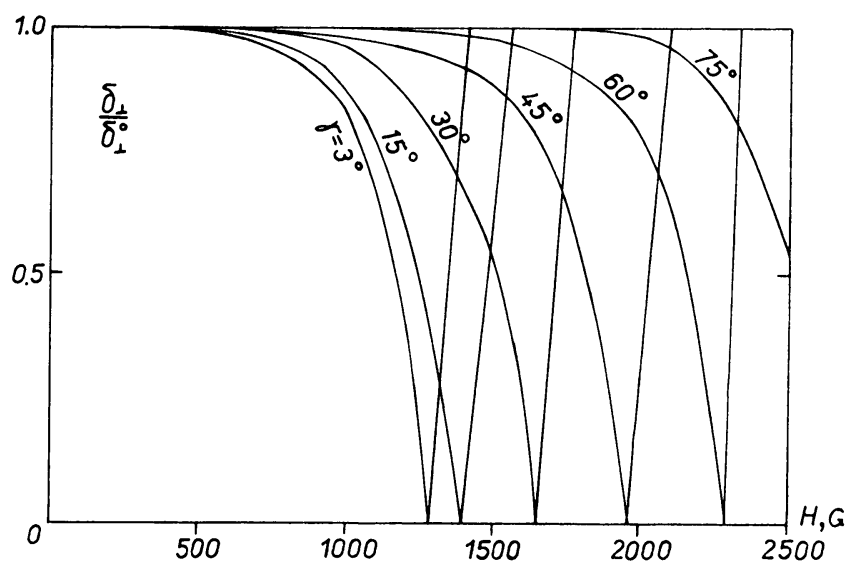

Fig. 3. The ratio $\delta_{\perp} / \delta_{\perp}{ }^{0}$ upon $H$ and $\gamma$ in mode $I I$.

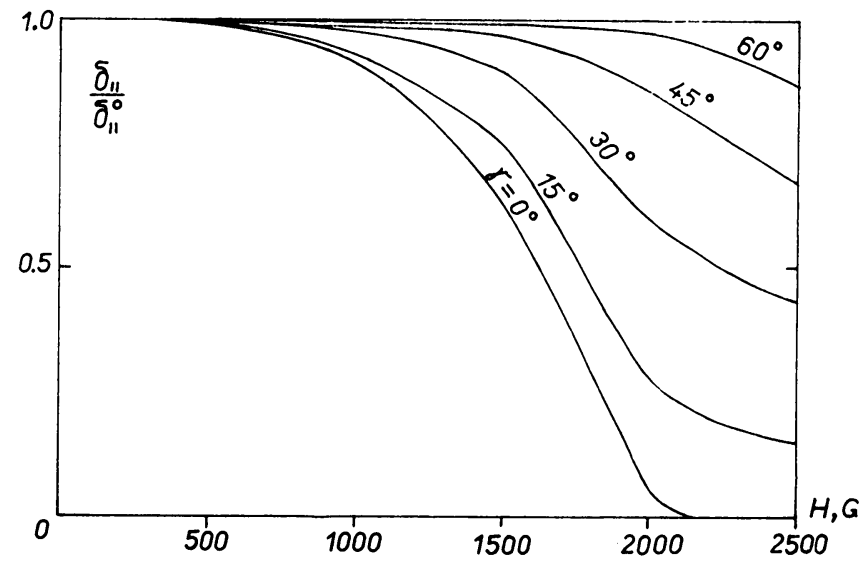

Fig. 4. The ratio $\delta_{\|} / \delta_{\|}{ }^{0}$ as a function of field strength $H$ and inclination $\gamma$ (mode $I I$ ).

from Figure 4, the relative errors of $\delta_{\|}$, in general, are smaller than those of $\delta_{\perp}$. When $H \approx 1500 \mathrm{G}$ errors do not exceed $10 \%$ for $\gamma>30^{\circ}$.

The calculation shows that the errors in azimuth can appear only in the first mode and they do not exceed $8^{\circ}$ for $H \approx 1500 \mathrm{G}$ and $\gamma>45^{\circ}$; when $H \lesssim 1000 \mathrm{G}$ errors are less than $8^{\circ}$ for arbitrary $\gamma$.

To summarize the results we conclude that the total vector measurements with a Crimean-type magnetograph are quite satisfactory for $H \lesssim 1000 \mathrm{G}$. For stronger 
fields the errors are acceptable if the inclination $\gamma \gtrsim 45^{\circ}$. When $H>1500 \mathrm{G}$ and $\gamma<45^{\circ}$ the miscentering effect leads to considerable errors, but for such strong fields magnetograph results, in general, are inadequate due to saturation. So the miscentering is only of importance when the longitudinal field $H_{\|}>1500 \mathrm{G}$. The second mode of the Crimean magnetograph has a smaller error than that of mode $I$.

One should note that all calculations of the magnetograph signal are usually based on some theory of the line formation in the presence of magnetic fields, and thus they

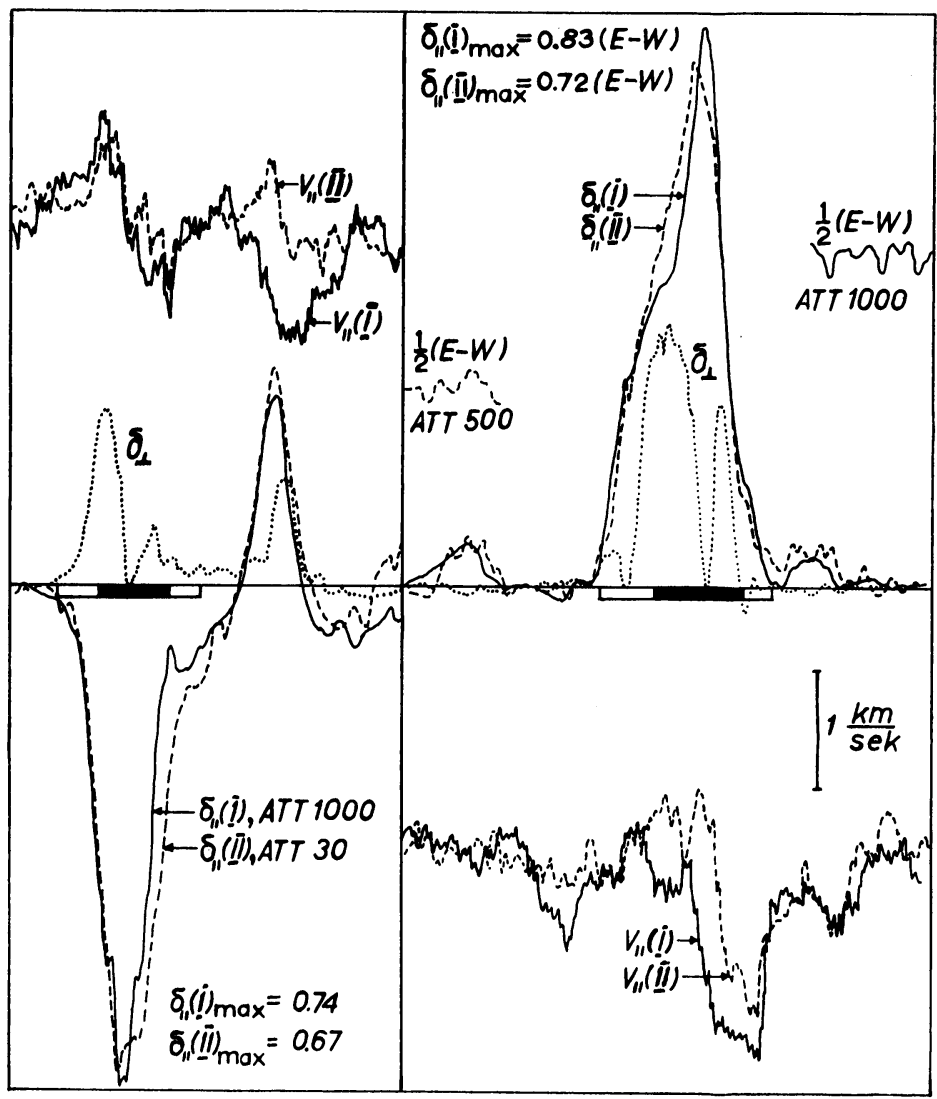

Fig. 5. The recordings of $\delta_{\|}, \delta_{\perp}$ and velocity signals obtained in a spot by both modes of the magnetograph. The value $E-W$ means the calibration signal.

are scarcely consistent in view of the simplifications inherent in theoretical models. The values of systematic errors found here and earlier by Wiehr (1969) are much in excess, because the numerous observations with our magnetograph give clear evidence that the fictitious velocities turned out to be considerably smaller than the calculated values. The most surprising result from Figure 5 is that the difference between maximum signals $\delta_{\|}$obtained in both modes is found to be smaller than $14 \%$ and the maximum fictitious velocity $\Delta V_{\|} \lesssim 1 \mathrm{~km} / \mathrm{s}$, while the field $H_{\|} \gtrsim 2000 \mathrm{G}$ in the spot. 
A number of investigations made with the magnetograph and using the photographic technique have shown that the field in the penumbra is mainly transversal $\left(\gamma>50^{\circ}\right)$; in the umbra often $\gamma>30^{\circ}$ and only in a very narrow region is the field purely longitudinal. These facts together with our calculations give a reason to consider the measurements with the Crimean vector magnetograph to be quite reliable up to field-strength $H_{\|} \approx 1500 \mathrm{G}$. Probably this value of $H_{\|}$can be much greater when the empirical calibration is applied. This follows also from the fact that results on the field structure in spots obtained in the $\lambda 5250$ line are confirmed by similar data obtained in spectral lines of small magnetic sensitivity, for which errors introduced by miscentering are almost negligible.

It should be stressed here that the miscentering effect cannot explain the discrepancy between theoretical and empirical calibration curves originally found by Severny (1967). This discrepancy is still not quite clear and should be considered in future research. It is possible that this discrepancy has the same origin as the discrepancy between calculated and observed miscentering effects that we have just described.

\section{References}

Beckers, J. M.: 1968, Solar Phys. 5, 15.

Kuznecov, D. A., Kuklin, G. V., and Stepanov, V. E.: 1966, Rezultaty nabljud. $i$ issled. $v$ period MGSS 1, 80.

Nikulin, N. S.: 1967, Izv. Krymsk. Astrofiz. Obs. 36, 76.

Severny, A. B.: 1967, Izv. Krymsk. Astrofiz. Obs. 36, 22.

Stepanov, V. E. and Severny, A. B.: 1962, Izv. Krymsk. Astrofiz. Obs. 28, 166.

Unno, W.: 1956, Publ. Astron. Soc. Japan 8, 108.

Wiehr, E.: 1969, Solar Phys. 9, 225.

\section{Discussion}

Schröter: Did you ever consider light retardation in your instrument, since if such an instrumental retardation exists you should in reality not observe in mode 1 and in mode 2 (the two positions of the $\lambda / 4$ plate) but somewhere in between. This very probably can explain why you are not observing the miscenterings as expected.

Severny: The 'retardation' properties of our solar tower (or better to say the instrumental elliptical polarization) have been considered in several papers: by Stepanov and Severny (1962, Publ. Crim. Obs.), by Severny (1964, Publ. Crim. Obs.). If the coatings of mirrors are not fresh and proper and we avoid oblique incidence the effect of retardation (due to reflection from aluminized surfaces) is not more than $0.2 \%$. The elliptical polarization due to scattered light inside a sunspot can easily be measured by using a magnetically insensitive line e.g. $\lambda 5123$, and this effect brings no more than $200 \mathrm{G}$ inside the umbrae of sunspots (with a field $\geqslant 1500 \mathrm{G}$ ). The effects of miscentering and the instrumental polarization are probably most appreciable in such cases as Coudé spectrographs like Locarno (and Oxford) because of the strong influence of changing the azimuth of polarization (rotation of the field of view). 